\title{
The research on the response of the structure and the load of gantry cranes cylindrical structures based on strain-measuring method
}

\author{
Jiang Aihua ${ }^{1, a^{*}}$,Deng Xianyuan ${ }^{1, b}$, Guo Zhongliang ${ }^{1, \mathrm{c}}$, ,He Shan ${ }^{1, \mathrm{~d}}$ \\ ${ }^{1}$ Research and development center, \\ Guangzhou Academy of Special Equipment Inspection \& Testing, Guangzhou, China \\ aaiwa9104@126.com, bdengxianyuan@gmail.com, 467096155@qq.com, dheshan321@163.com
}

Keywords: Gantry cranes, Cylindrical structures, Strain-measuring

\begin{abstract}
In this paper, a shipbuilding gantry crane serviced for up to 18 years is studied; the strain-measuring method is used for testing and analyzing the response of the structure and the load about the main force component-cylindrical structural. By arranging the measuring point $90^{\circ}$ to each other on the cylindrical section of the maximum bending moment, the rule of stress conditions of cylinder is established. The results showed that: when the crane lifting, the same side in the cylinder has the maximum compressive stress, which mapped side has the maximum tensile stress, the stress of both sides of the vertical part is minimum; When the rotating mechanism run a week, the same section of the cylinder will show a cyclical linear change with the rotation angle of the boom (jagged cyclical changes).
\end{abstract}

\section{Introduction}

In recent years, with the development of the shipbuilding industry, large-tonnage portal cranes serve as a pivotal role in the process wharf cargo handling, and performance requirements and scope of the lifting equipment is also increasing [1-3]. Due to limited slewing bearing load capacity, so cylindrical gantry crane has been widely used, the cylinder is important force component of cylindrical gantry crane, supporting the entire weight of the upper rotating part and all foreign load, the stability of the portal crane has important significance, so as to ensure the smooth operation of crane, the cylinder must have sufficient strength, in particular to have greater stiffness [4. 5]. In this paper, the strain measuring method of cylindrical structure and load response were tested to study the suspended load variation, the portal crane cylinder design and safety inspection has important guiding significance.

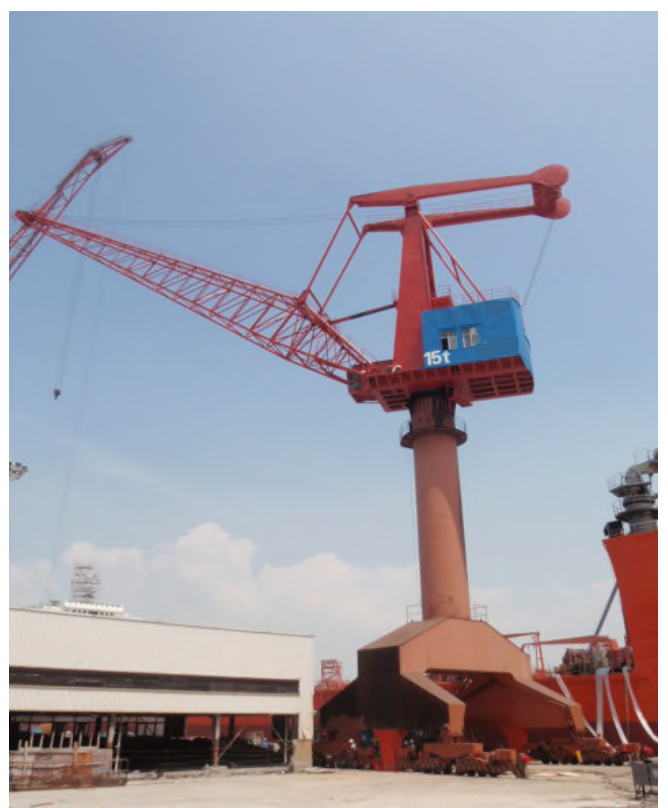

Fig. 1 Testing sample a testing points. 
Object of this test is a 18-year service shipbuilding portal crane, rated load $15 \mathrm{t}$, the maximum amplitude is $48 \mathrm{~m}$, and the minimum amplitude is $23 \mathrm{~m}$, Fig. 1 shows the test sample, the measuring points are selected on the cross section of the maximum bending, Test equipment used primarily to testing is TMR-200 multi-channel data acquisition system, the strain gauges are FLA-5-11-1L-type, which the sensitivity coefficient $\mathrm{K}$ is $2.11 \pm 1 \%$.the resistance value $\mathrm{R}$ is $119.8 \pm 0.5 \Omega$, the sampling frequency is set to $50 \mathrm{~Hz}$.

\section{Data Analysis and Results of the Discussions}

Instrument data is cleared when shipbuilding portal crane stayed at the minimum amplitude, then started recording, the curves of the tested strain and time can obtained. Since the testing is carried out within the elastic range of the structure, according to the generalized Hooke's law, the final stress - time curves that shown in Fig. 2 - Fig. 3 can obtained. Abscissa is time and the vertical coordinate is the measured stress.

Table 1. Related actions timetable.

\begin{tabular}{c|c|c|c|c}
\hline time & $0^{\prime \prime}$ & $178^{\prime \prime}$ & $208^{\prime \prime}$ & $310^{\prime \prime}$ \\
\hline action & 10 tifed loads & up brake & change 48m & Stay $30^{\prime \prime}$ \\
\hline time & $397^{\prime \prime}$ & $482^{\prime \prime}$ & $517^{\prime \prime}$ & $581^{\prime \prime}$ \\
\hline action & $\begin{array}{c}\text { Counterclockwise } \\
\text { rotation of } 90^{\circ}\end{array}$ & Stay $30^{\prime \prime}$ & $\begin{array}{c}\text { Counterclockwise } \\
\text { rotation of } 90^{\circ}\end{array}$ & Stay $30^{\prime \prime}$ \\
\hline time & $630^{\prime \prime}$ & $688^{\prime \prime}$ & $720^{\prime \prime}$ & $798^{\prime \prime}$ \\
\hline time & $\begin{array}{c}\text { Clockwise } \\
\text { rotation of } 90^{\circ}\end{array}$ & Stay $30^{\prime \prime}$ & $\begin{array}{c}\text { Clockwise } \\
\text { rotation of } 90^{\circ}\end{array}$ & Stay $30^{\prime \prime}$ \\
\hline action & $\begin{array}{c}\text { Clockwise } \\
\text { rotation of } 90^{\circ}\end{array}$ & Stay $30^{\prime \prime}$ & $\begin{array}{c}\text { Counterclockwise } \\
\text { rotation of } 90^{\circ}\end{array}$ & change 23m \\
\hline
\end{tabular}

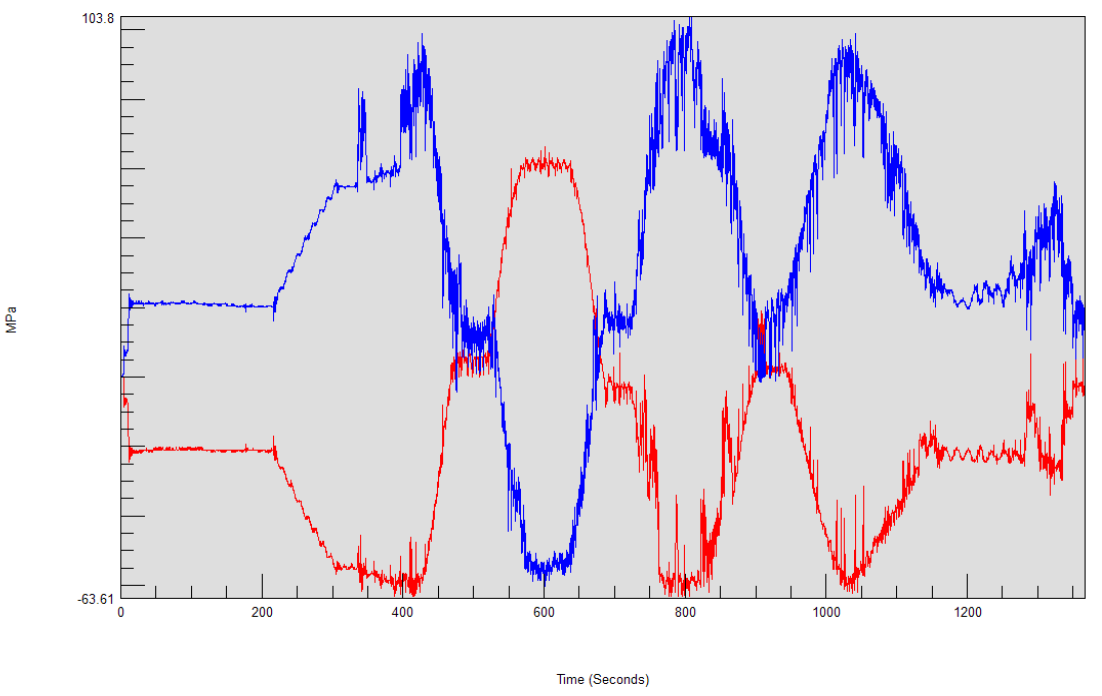

Fig. 2 The testing results of measuring points 1 and 3. 


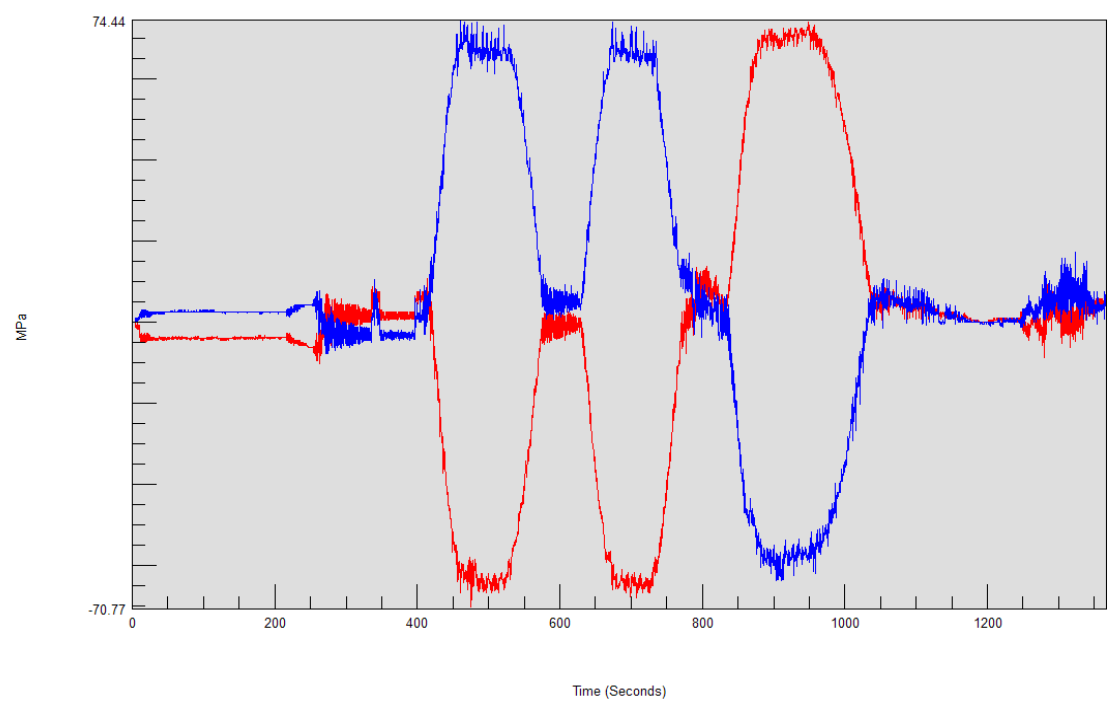

Fig. 3 The testing results of measuring points 2 and 4.

According to the above data, when the measuring point and the boom are the same side, the force is greater, and the back has approximately tensile stress, with a $90^{\circ}$ to the measuring point has a smaller force; when rotary mechanism is rotating, the stress of the measuring point on the cylinder changes immediately [6-8].

According to Fig. 3, the change of the stress curve when the rotary mechanism is rotated can be found: the stress value and the rotation angle linear. Case in measuring point 2 , the definition of the boom and measuring point 2 which stays on the same side is 0 degrees, the linear relationship between the measuring point and the angle of rotation when the rotary mechanism counterclockwise rotation is analyzed, as shown in Table 2.

Table 2. The stress of measuring point 2 when boom rotation.

\begin{tabular}{ccccc}
\hline $\begin{array}{c}\text { angle of boom } \\
\text { rotation }\end{array}$ & $0^{\circ}$ & $90^{\circ}$ & $180^{\circ}$ & $270^{\circ}$ \\
\hline Stress (MPa) & -66.5 & -1.0 & 69.5 & 1.49 \\
\hline
\end{tabular}

Seen from Table 2, the angle of the measuring point 2 and the boom is from $0^{\circ}$ to $180^{\circ}$, the forces are on the opposite direction, but have approximately equal size, field testing errors and other are considered, to facilitate the analysis, the absolute mean value is now as the size of the force, i.e. $68 \mathrm{MPa}$; Similarly, the angle of the measuring point 2 and boom is $90^{\circ}$ or $270^{\circ}$, the force is taked 0MPa. Fig. 4 is curve of stress and rotating angle of measuring point 2.

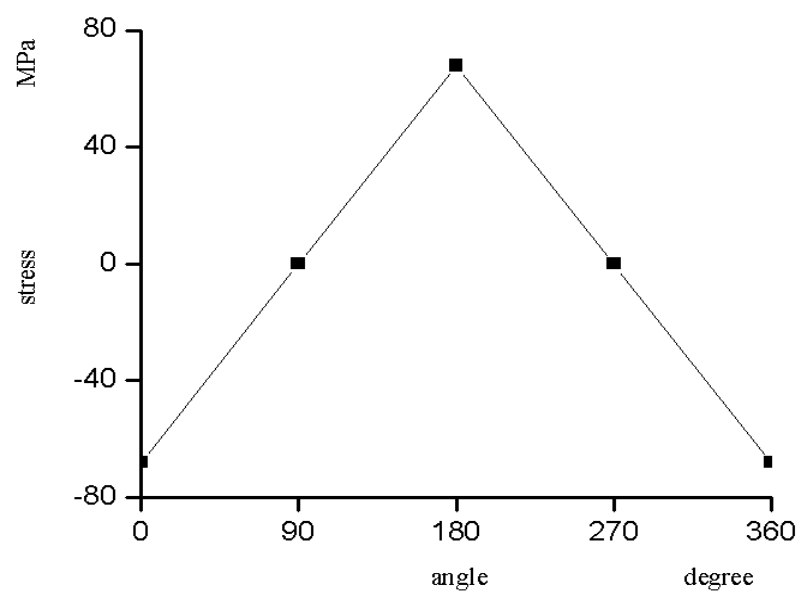

Fig. 4 The curve of the stress and rotation angle of point 2. 
Seen from Figure 4, the curve of the force and the rotational angle of the measuring point 2 are changed periodically jagged. When the counterclockwise rotation angle from $0^{\circ}$ to $180^{\circ}$, the force of measuring points 2 increases with the force of the rotation angle increased, the size of the angle $\alpha$ and $\mathrm{F}$ can be expressed as the relation:

$$
F=\left(\frac{\alpha}{90}-1\right) 68
$$

When the rotation angle counterclockwise from $180^{\circ}$ to $360^{\circ}$, the force of the measuring points 2 increases with the rotation angle of the force decreased, the size of the angle $\alpha$ and $\mathrm{F}$ can be expressed as the relation:

$$
F=\left(3-\frac{\alpha}{90}\right) 68
$$

Promoting Formula 1 and Formula 2 shows that the relationship of the rotary mechanism can be repressed in the case of the measured force size and structure of the boom cylinder of portal crane for cylindrical structures.

$$
\begin{aligned}
& F=\left(\frac{\alpha}{90}-1\right) Q \quad\left(0 \leq \alpha \leq 180^{\circ}\right) \\
& F=\left(3-\frac{\alpha}{90}\right) Q \quad\left(180^{\circ}<\alpha \leq 360^{\circ}\right)
\end{aligned}
$$

Through the testing of the cylinder structure of portal crane, comparing the test results of type 3 and type 4, according to the data of the cylindrical structure whether there is a security risk, such as the symmetry of the structure of the door frame, the level degree of tracks, the rigidity of cylinder.

\section{Conclusion}

After the testing of the cylindrical structure of the gantry cranes, the testing results shows that the stress of the measurement point on the cylinder changes with the rotation of the rotary body, and periodically jagged curve; Further, during the rotation of the cylinder the force change and a linear relationship between the angle of rotation can be used to guide the design and safety assessment cylindrical structure.

\section{Acknowledgements}

This work was financially supported by Technology Projects of Guangdong Provincial Quality and Technical Supervision (2015CT02、2015CT09、2016CT05), Guangzhou Innovation Platform and Sharing Special Projects (201509010008), Science and Technology Planning Project of Guangdong Province (2014a040401046).

\section{References}

[1] H. Y. Chen, L. B. Zhu, X. Huang, Y. H. Zhang. Structural Health Monitoring System of Gantry Crane Based on ZigBee Technology. 2012 Third International Conference on Digital Manufacturing \& Automation, (2012), pp. 801-804.

[2] Y. C. Fan, Research on Wireless Remote Monitoring System to Metal Structural Stresses on Port Crane, Port Operat. (3) (2011) (in Chinese)

[3] F. G. Zhang, Study on the short range wireless communication technique and its merging developing trends, Electr. Measure. Instrument. (10) (2007) (in Chinese). 
[4] W. Pei, Research of Gantry Crane Health Monitoring Experiment System Based on Option Fiber Grating, Master Degree thesis. Taiyuan, Shanxi, China: North University of China. 2010. 4. (in Chinese)

[5] S. Rajeev, Ananda. Mobile, Wireless and Sensor Networks. John Wiley \& Sons, Inc, USA, 2006.

[6] China Communications Standards Association, ZigBee Technique and its Applications, 2007. (in Chinese)

[7] W. Chen, Study on Maintenance Decision Support System of Crane Based on Online Monitoring, Master Degree thesis. Wuhan, Hubei, China: Wuhan University of Technology. 2010. 4. (in Chinese)

[8] H. M. Dong, D. F. Chen, S. J. Zou. Development framework for gantry crane training system based on virtual reality. The $2^{\text {nd }}$ international symposium on digital manufacturing. (10) (2009) 27-30. 